Pesq. Vet. Bras. 29(7):563-568, julho 2009

\title{
Doenças parasitárias em ruminantes no semi-árido brasileiro ${ }^{1}$
}

\author{
Valéria Medeiros de M. Costa ${ }^{2}$, Sara V.D. Simões ${ }^{2}$ e Franklin Riet-Correa ${ }^{2^{*}}$
}

\begin{abstract}
Costa V.M.M., Simões S.V.D. \& Riet-Correa F. 2009. [Parasitic diseases in ruminants in the Brazilian semiarid.] Doenças parasitárias em ruminantes no semiárido brasileiro. Pesquisa Veterinária Brasileira 29(7):563-568. Hospital Veterinário, CSTR, Universidade Federal de Campina Grande, Campus de Patos, 58700-970 Patos, PB, Brazil. Email: franklin.riet@pq.cnpq.br

Clinical cases and diagnostic specimens from ruminants received by the Veterinary Hospital of the University of Campina Grande in Patos, Paraíba, Brazil, from January 2000 to August 2007, were reviewed to evaluate some epidemiological aspects of parasitic diseases of ruminants in the semiarid region of Paraíba and neighboring states. The region has an annual rainfall of about $800 \mathrm{~mm}$, with irregular rains concentrated in a 3 to 4 -month-period, and an average annual temperature of $26^{\circ} \mathrm{C}$. During those years, $163(5.31 \%)$ out of 3,064 ruminants were affected by some parasitic disease. The most important parasitosis in goats and sheep was gastrointestinal helminthiasis, mainly hemonchosis; goats were more affected $(6.24 \%$ of cases in this species) than sheep ( $4.7 \%$ of cases). The higher frequency of the disease in goats may be associated with higher susceptibility or due to treatment mistakes, i.e. use of the same dose for both species, which for most anti-antihelmintics is insufficient for goats. Cattle have a low rate of gastrointestinal helminthiasis (1 out of 1,113 cases). This low frequency is probably due to the farming system in the semiarid, with low stocking rate, up to one adult bovine for every 13-16 ha, and permanence of calves (susceptible) with their mothers (resistant) for nearly one year before weaning. The occurrence of eimeriosis in goats and sheep was observed in $0.76 \%$ of the cases, involving only young animals. The main cattle disease was tick fever with 14 outbreaks. Outbreaks of tick fever occur, mainly at the end of the raining season in areas of the semiarid which are marginal for tick, like plateaus and mountains of the Borborema region, irrigated areas, and areas of the basins of two rivers of the region. In the drier areas of the semiarid Rhipicephalus (Boophilus) microplus do not survive during the dry period, but tick fever can occur when cattle with ticks are introduced at the onset of the raining season.
\end{abstract}

INDEX TERMS: Ruminant, parasitic diseases, helminthiasis, parasitosis, tick fever.

RESUMO.- Arquivos de fichas clínicas de ruminantes ou amostras para diagnósticos recebidos pelo Hospital Veterinário da Universidade de Campina Grande, em Patos, Paraíba, de janeiro 2000 a agosto 2007, foram revisados para avaliar alguns aspectos epidemiológicos de doen-

\footnotetext{
${ }^{1}$ Recebido em 7 de novembro de 2008.

Aceito para publicação em 11 de março de 2009.

${ }^{2}$ Hospital Veterinário, CSTR, Universidade Federal de Campina Grande, Campus de Patos, 58700-970 Patos, PB, Brasil. *Autor para correspondência; E-mail: franklin.riet@pq.cnpq.br
}

ças parasitárias de ruminantes na região semi-árida da Paraíba e estados vizinhos. A região apresenta precipitações médias anuais de aproximadamente $800 \mathrm{~mm}$, com chuvas irregulares concentradas em um período de 3-4 meses e uma temperatura média anual de $26^{\circ} \mathrm{C}$. No período, $163(5,31 \%)$ de 3.064 ruminantes foram afetados por alguma doença parasitária clinica. A doença parasitária mais frequente em caprinos e ovinos foi a helmitose gastrointestinal, principalmente a hemonchose, e caprinos foram mais afetados $(6,24 \%$ dos casos diagnosticados) do que os ovinos (4,7\% dos casos diagnosticados). 
A maior freqüência da doença nos caprinos pode estar associada à maior susceptibilidade destes ou devido a erros no tratamento, como a utilização da mesma dose para as duas espécies, o que para a maioria dos antiantihelmínticos é insuficiente para caprinos. Bovinos tiveram uma baixa freqüência de helmintoses gastrintestinais (1 de 1.113 casos). Esta baixa freqüência deveu-se, provavelmente, ao sistema de criação no semi-árido, com baixa taxa de lotação, até um animal adulto por hectare a cada 13-16 hectare, e a permanência de bezerros (suscetíveis) com as mães (resistentes) por períodos de até um ano antes da desmama. A ocorrência de eimeriose em caprinos e ovinos foi de $0,76 \%$ dos casos, envolvendo apenas os animais jovens. Em bovinos a principal doença foi à tristeza parasitária com 14 surtos. Os surtos de tristeza ocorreram principalmente no final do período chuvoso em áreas de desequilíbrio enzoótico incluindo as montanhas e planaltos da região da Borborema, áreas irrigadas, e áreas das bacias do Rio do Peixe e Rio Piranhas. Nas áreas mais secas do semi-árido o Rhipicephalus (Boophilus) microplus não sobrevive durante o período de seca, mas, tristeza parasitária pode ocorrer quando no início do período chuvoso bovinos com carrapatos são introduzidos e esses se multiplicam durante o mesmo.

TERMOS DE INDEXAÇÃO: Ruminantes, enfermidades parasitárias, semi-árido, verminose, parasitoses, tristeza parasitária.

\section{INTRODUÇÃO}

Em ruminantes, as doenças parasitárias são responsabilizadas por elevadas perdas econômicas em decorrência de crescimento retardado, perda de peso, redução no consumo de alimentos, queda na produção de leite, baixa fertilidade e nos casos de infecções maciças, altas taxas de mortalidade, além de custos para o seu controle (Vieira 1999).

A elevada prolificidade, adaptabilidade e resistência a diversas condições climáticas fazem com que tanto ecto quanto endoparasitas tenham ampla distribuição geográfica e alta prevalência, tanto em regiões com clima temperado como clima tropical. Sabe-se que cada parasita possui um determinado número de combinações ecológicas que permitem seu desenvolvimento em uma determinada região e não em outra (Molento 2005). A maior ou menor prevalência de uma ou mais espécies depende de um conjunto de fatores como: temperatura, precipitação pluviométrica, solo, tipo e manejo da pastagem, espécie, raça, idade, estado fisiológico e nutricional e manejo dos animais (Ruas \& Berne 2001). Desta forma, o conhecimento das doenças parasitárias com suas particularidades regionais é indispensável para a formulação de programas eficientes de controle. $O$ objetivo deste trabalho foi avaliar pela analise do histórico das fichas clínicas e amostras enviadas para diagnóstico, alguns aspectos epidemiológicos das doenças parasitárias mais comuns em ruminantes no semi-árido da Paraíba e Estados vizinhos, no período de janeiro de 2000 a agosto de 2007 .
Com esta informação é possível determinar formas mais eficientes de controle e, conseqüentemente, diminuir as perdas ocasionadas pelas parasitoses.

\section{MATERIAL E MÉTODOS}

Foram revisadas, nos arquivos do Laboratório de Patologia Animal (LPA) e Clínica de Grandes Animais (CGA) do Hospital Veterinário (HV) de Patos da Universidade Federal de Campina Grande (UFCG), as fichas dos animais atendidos nestes setores no período janeiro de 2000 a agosto de 2007, identificando-se os casos de enfermidades parasitárias clínicas diagnosticadas em ruminantes.

Os animais atendidos no HV são provenientes de três estados: Paraíba, Pernambuco e Rio Grande do Norte. Os municípios de origem dos animais fazem parte de região semi-árida, com precipitações médias anuais iguais ou inferiores a $800 \mathrm{~mm}$, temperatura média anual de $26^{\circ} \mathrm{C}$ e um regime de chuvas marcado pela escassez, irregularidade e concentração das precipitações num curto período, de apenas 3-4 meses (Adene 2007).

Foi realizado o levantamento de dados referentes à idade, época de ocorrência das doenças, e sinais clínicos observados, considerando todos os surtos ou casos isolados cujos diagnósticos haviam sido confirmados pelos sinais clínicos, necropsias realizadas e/ou exames complementares tais como o parasitológico de fezes e raspado de pele.

\section{RESULTADOS}

No período de janeiro de 2000 a agosto de 2007, foram atendidos 3.064 ruminantes no HV, dos quais $163(5,31 \%)$ estavam acometidos por alguma enfermidade parasitária. O percentual de diagnósticos de doenças parasitárias para cada espécie pode ser observado no Quadro 1.

No Quadro 2 estão as principais doenças parasitárias diagnosticadas no HV e seus respectivos percentuais de ocorrência. Dois casos de sarna foram diagnosticados em caprinos, sendo um causado por Demodex caprae e outro por Chorioptes bovis. Neste último, os sinais observados foram prurido intenso, áreas alopécicas com crostas no dorso e descamação na face interna da coxa, nas quartelas, na face caudal do escroto, abdômen e axila.

Os casos de miíase ocorridos em caprinos e ovinos foram descritos por Macêdo et al. (2008). Àqueles observados em bovinos ocorreram em animais adultos, e as lesões foram vistas na região umbilical e na vulva.

Dos 111 casos de verminose encontrados, 63 (47 em caprinos, 16 em ovinos) foram diagnosticados como hemoncose, onde 31 (22 caprinos, 9 ovinos) tiveram seu

\section{Quadro 1. Surtos das doenças parasitárias de ruminantes atendidos no Hospital Veterinário, Universidade Federal de Campina Grande, no período de janeiro de 2000 a agosto de 2007}

\begin{tabular}{lcc}
\hline Espécies & $\begin{array}{c}\text { Atendidos } \\
\mathrm{N}\end{array}$ & $\begin{array}{c}\text { Com doenças parasitárias } \\
\mathrm{N}(\%)\end{array}$ \\
\hline Caprinos & 1186 & $101(8,5 \%)$ \\
Ovinos & 765 & $44(5,7 \%)$ \\
Bovinos & 1113 & $18(1,6 \%)$ \\
Total & 3064 & $163(5,3 \%)$
\end{tabular}


Quadro 2. Parasitoses diagnosticadas em ruminantes atendidos no Hospital Veterinário, Universidade Federal de Campina Grande, no período de janeiro de 2000 a agosto de 2007

\begin{tabular}{|c|c|c|c|c|c|c|c|c|}
\hline \multirow[t]{2}{*}{ Doenças } & \multicolumn{2}{|c|}{ Caprinos } & \multicolumn{2}{|c|}{ Bovinos } & \multicolumn{2}{|c|}{ Ovinos } & \multicolumn{2}{|c|}{ Total } \\
\hline & $\mathrm{N}$ & $\%^{a}$ & $\mathrm{~N}$ & $\%^{a}$ & $\mathrm{~N}$ & $\%^{a}$ & $\mathrm{~N}$ & $\%$ \\
\hline Sarna & 2 & 0,17 & & - & . & & 2 & 0,06 \\
\hline & 4 & & 2 & 0,18 & 1 & 0,14 & 8 & 0,23 \\
\hline & 18 & & 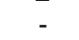 & - & 5 & & 23 & 0,76 \\
\hline & 74 & 6,24 & 1 & 0,09 & 36 & 4,70 & 111 & 3,62 \\
\hline & 3 & 0,25 & 1 & 0,09 & 2 & 0,26 & 6 & 0,19 \\
\hline Trist & - & - & 14 & 1,26 & - & - & 14 & 0,46 \\
\hline Outras doenças & 1085 & 91,48 & 1095 & 98,38 & 721 & 94,25 & 2901 & 94,68 \\
\hline Total & 1186 & 100 & 1113 & 100 & 765 & 100 & 3064 & 100 \\
\hline
\end{tabular}

a Percentual em relação ao total de casos nessa espécie.

b Parasitismo por verminose associado à eimeriose.

diagnóstico confirmado pela necropsia, sendo observados numerosos parasitas adultos de Haemonchus contortus no abomaso em todos os animais necropsiados. Em outros 32 casos (25 em caprinos, 7 em ovinos), que se recuperaram, foi diagnosticado hemoncose com base na presença de sinais clínicos característicos (palidez de mucosas e edema submandibular) e exame coprológico (contagem de ovos por g de fezes, OPG). Os demais casos (27 em caprinos, 20 em ovinos, 1 em bovinos) foram diagnosticados como verminose, sem especificar as espécies de nematódeos envolvidas, com base nos dados de epidemiologia e exame coprológico com determinação de OPG (Quadro 2).

O diagnóstico da eimeriose foi realizado com base nos sinais clínicos e no exame coprológico dos animais (5 animais ) e da necropsia feita em 18 casos (16 em caprinos e 2 em ovinos). Todos os casos de eimeriose ocorreram em animais jovens com 15 dias a 11 meses idade (Quadro 2).

O percentual de animais por categoria (jovens e adultos) parasitados nas três espécies pode ser observado no Quadro 3. Observa-se que a parasitose gastrintestinal de caprinos e ovinos afeta igualmente adultos e jovens, enquanto que a coccidiose afeta animais jovens. Em bovinos a tristeza parasitária afeta principalmente adultos.

Na Figura 1 observa-se a ocorrência mensal das enfermidades parasitárias em caprinos, no período de ja-

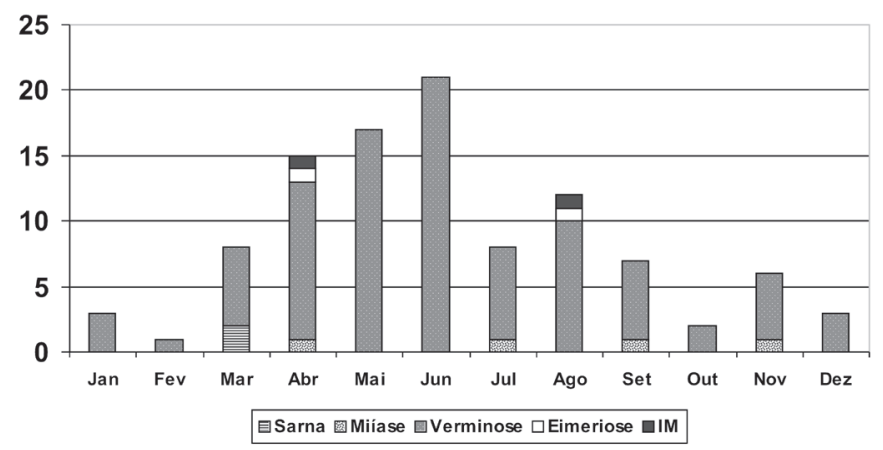

Fig.1. Número de surtos de doenças parasitárias nos anos de 2000-2007 por mês, em caprinos na Paraíba. IM = infecção mista (eimeriose e verminose).

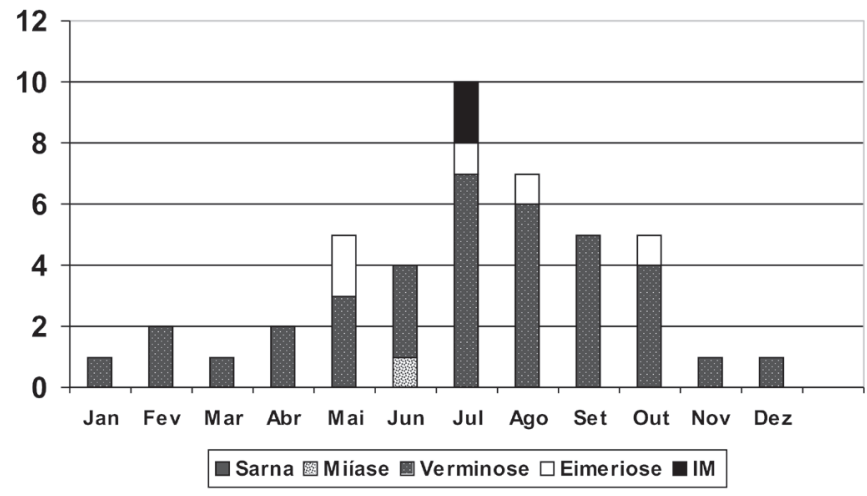

Fig.2. Número de surtos de doenças parasitárias nos anos de 2000-2007 por mês, em ovinos Paraíba. IM = infecção mista (eimeriose e verminose).

neiro de 2000 a abril de 2007. Observa-se um número crescente de surtos de parasitoses gastrintestinais entre os meses de março a junho, na medida em que avança o período de chuvas, e com a maior freqüência no final das chuvas e início da seca.

Na Figura 2 observa-se a ocorrência mensal das enfermidades parasitárias em ovinos, no período de janeiro de 2000 a abril de 2007. Em ovinos o maior número de surtos de parasitoses gastrintestinais também se constata nos meses de final das chuvas e inicio da seca (maio-junho).

Na Figura 3 observa-se a ocorrência mensal das enfermidades parasitárias em bovinos, no período de janei-

Quadro 3. Freqüência de caprinos, ovinos e bovinos parasitados de acordo com a categoria

\begin{tabular}{|c|c|c|c|c|c|c|c|c|c|c|c|c|}
\hline \multirow[t]{2}{*}{ Doenças } & \multicolumn{4}{|c|}{ Caprino } & \multicolumn{4}{|c|}{ Ovino } & \multicolumn{4}{|c|}{ Bovino } \\
\hline & $\begin{array}{c}\mathrm{Jo}^{\mathrm{a}} \\
\%\end{array}$ & $\begin{array}{c}\mathrm{Ad}^{\mathrm{b}} \\
\%\end{array}$ & $\begin{array}{c}\mathrm{NI}^{\mathrm{C}} \\
\%\end{array}$ & $\begin{array}{c}\text { Total } \\
\mathrm{N}\end{array}$ & $\begin{array}{c}\mathrm{Jo}^{\mathrm{a}} \\
\%\end{array}$ & $\begin{array}{c}\mathrm{Ad}^{\mathrm{b}} \\
\%\end{array}$ & $\begin{array}{l}\mathrm{NIC} \\
\%\end{array}$ & $\begin{array}{c}\text { Total } \\
\mathrm{N}\end{array}$ & $\begin{array}{c}\mathrm{Jo}^{\mathrm{a}} \\
\%\end{array}$ & $\begin{array}{c}\mathrm{Ad}^{\mathrm{b}} \\
\%\end{array}$ & $\begin{array}{c}\mathrm{NI}^{\mathrm{C}} \\
\% \\
\end{array}$ & $\begin{array}{c}\text { Total } \\
\text { N }\end{array}$ \\
\hline Sarna & - & 100 & - & 2 & - & - & - & - & - & - & - & - \\
\hline Miíase & & 50 & 50 & - & 4 & - & 100 & - & $1-$ & 100 & - & 2 \\
\hline Verminose & 33.78 & 62.16 & 4.05 & 74 & 52.78 & 47.22 & - & 36 & 100 & - & - & 1 \\
\hline Eimeriose & 100 & - & - & 18 & 100 & - & - & 5 & - & - & - & - \\
\hline $\mathrm{IM}^{\mathrm{d}}$ & 33.33 & 33.33 & 33.33 & 3 & 50 & 50 & - & 2 & 100 & - & - & 1 \\
\hline TPB & - & - & - & - & - & - & - & - & 20 & 73.33 & 6.67 & 14 \\
\hline Total & 46 & 51 & 4 & 101 & 25 & 19 & - & 44 & 5 & 12 & 1 & 18 \\
\hline
\end{tabular}

a Jo = animais jovens, com menos de 1 ano de idade, ${ }^{b} \mathrm{Ad}=$ animais com mais de 1 ano de idade,

${ }^{c} \mathrm{NI}=$ não informado, ${ }^{d} \mathrm{IM}$ infecção mista. 


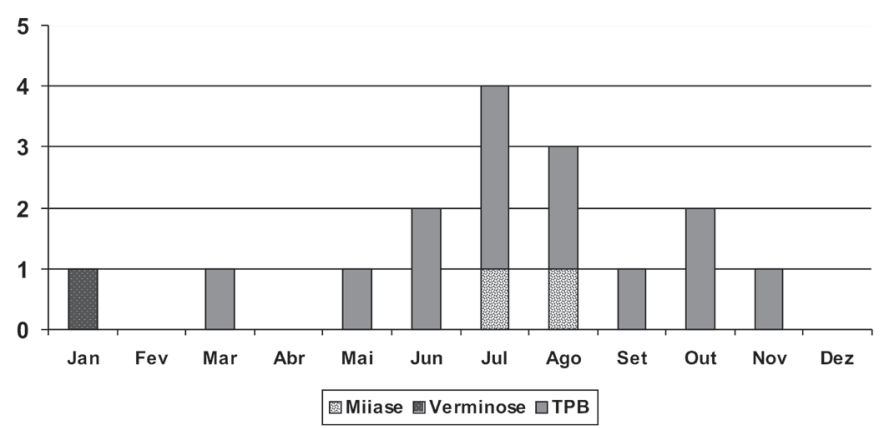

Fig.3. Número de surtos de doenças parasitárias nos anos de 2000-2007 por mês, em bovinos na Paraíba. TPB = Tristeza parasitária.

Quadro 4. Dados epidemiológicos dos surtos de tristeza parasitária, anaplasmose e babesiose diagnosticados em bovinos no Hospital Veterinário, Universidade Federal de Campina Grande, por faixa etária, mês e ano de ocorrência e município de origem dos animais

\begin{tabular}{ccccc}
\hline Diagnóstico & Faixa etária & Mês & Ano & Município \\
\hline$A^{a}$ & 6 anos & Maio & 2006 & Patos \\
$A^{a}$ & 5 anos & Julho & 2006 & Patos \\
$A^{a}$ & NI d & Julho & 2002 & NI d \\
$A^{a}$ & 2 anos & Novembro & 2004 & S. J. Bonfim \\
$A^{a}$ & 2 meses & Agosto & 2006 & Patos \\
$B^{b}$ & 3 anos & Junho & 2002 & Catingueira \\
$B^{b}$ & 2 anos & Julho & 2006 & S. J. Bonfim \\
TPB $^{\mathrm{a}}$ & 4 anos & Setembro & 2004 & Mãe d'água \\
TPB $^{c}$ & 6 anos & Outubro & 2006 & Patos \\
TPB $^{c}$ & 4 meses & Outubro & 2006 & Patos \\
TPB $^{c}$ & 6 anos & Janeiro & 2007 & S. J. Bonfim \\
TPB $^{c}$ & 5 meses & Agosto & 2002 & Santa Maria \\
TPB $^{c}$ & 3 anos & Junho & 2003 & NI \\
TPB $^{\mathrm{d}}$ & 4 anos & Março & 2007 & Santa Teresinha \\
\hline
\end{tabular}

a Anaplasmose (Anaplasma. marginale); ${ }^{\mathrm{b}}$ babesiose (Babesia spp.); ${ }^{\mathrm{c}}$ complexo babesiose e anaplasmose; d não informado.

ro de 2000 a abril de 2007. Observa-se que a doença mais freqüente é a tristeza parasitária, com maior número de surtos no final das chuvas, quando aumenta a população de Rhipicephalus (Boophilus) microplus. Um só surto de verminose gastrintestinal foi diagnosticado em bovinos no período.

No Quadro 4 observa-se a o mês e o município de ocorrência da tristeza parasitária em bovinos, no período de janeiro de 2000 a abril de 2007, e a idade dos animais afetados. A doença afeta principalmente animais adultos em diversas épocas do ano, mas principalmente nos meses de junho e julho.

\section{DISCUSSÃO}

Neste trabalho constatou-se que a parasitose mais importante em caprinos e ovinos é a parasitose gastrintestinal e que os caprinos (6,24\% dos diagnósticos) são mais afetados que os ovinos (4,7\% dos diagnósticos; Quadro 2, Fig.1 e 2). Por outro lado, os bovinos são muito pouco afetados por parasitoses gastrintestinais (Quadro 2, Fig. 3). A maior freqüência da doença em caprinos do que em ovinos pode estar associada ao hábito alimentar desses animais que, por preferirem forrageiras arbustivas, não foram expostos durante sua domesticação a altas infestações parasitárias (Costa Júnior et al. 2005). Em conseqüência da menor habilidade dos caprinos em desenvolver uma resposta imune contra os nematódeos, quando ambas as espécies pastejam gramíneas em forma conjunta, os caprinos são mais sensíveis do que os ovinos as infestações parasitárias (Torres-Acosta \& Hoste 2008). Em surtos diagnosticados de gastrenterite parasitária em rebanhos mistos de caprinos e ovinos é freqüente que ocorram sinais clínicos somente nos caprinos (Costa 2008). Outro aspecto responsável por essa maior incidência da doença em caprinos é que esta espécie é tratada com anti-helmínticos em forma semelhante aos ovinos, quando o correto para a maioria dos antihelmínticos seria tratar os caprinos com doses maiores (Torres-Acosta \& Hoste 2008). Este fato deve-se a que os caprinos metabolizam mais rapidamente os benzimidazóis e as lactonas macrocíclicas, diminuindo o tempo em que a droga permanece no sangue em níveis tóxicos para os parasitos, permitindo que um número maior de parasitos sobreviva (Csiro 1994).

O elevado número de casos de hemonchose diagnosticado em caprinos e ovinos neste estudo mostra a importância que tem Haemonchus contortus para a criação de pequenos ruminantes. Levantamentos realizados revelam que mais de $80 \%$ da carga parasitária de caprinos é composta por Haemonchus contortus (Costa \& Vieira 1984, Arosemena et al. 1999). Este parasita é responsável por uma elevada intensidade de infecção, levando os animais a um quadro clínico severo de anemia, devido a sua ação hematófaga, o que faz desse helminto o maior causador de perdas produtivas e aumento no custo da criação. As perdas econômicas são decorrentes da baixa produtividade, geralmente observada no período seco, e da alta mortalidade, que ocorre principalmente na estação chuvosa (Charles 1989). A importância da hemoncose no semi-árido faz com que as práticas de controle devam ser focadas nesta espécie, levando em consideração as suas características epidemiológicas e o alto risco de seleção de estirpes resistentes, fato já comprovado na região (Athayde et al. 1996, Rodrigues et al. 2007).

Outro fato que deve ser levado em conta no controle das helmintoses gastrintestinais em caprinos e ovinos é a maior freqüência de surtos entre os meses de maio e agosto (Fig.1 e 2), no final do período de chuvas e inicio da seca. Tratamentos anti-helmínticos, antes desse período crítico, poderão evitar esses surtos devidos à contaminação crescente das pastagens durante o período de chuvas.

A baixa freqüência de parasitismo gastrintestinal nos bovinos (Quadro 2) se deve, provavelmente, às características da criação desses animais no semi-árido, com baixa lotação, de até uma unidade animal para cada 1316 hectares, e permanência dos bezerros (susceptíveis) com as mães (resistentes) por período longo, de um ano ou mais; com isto a lotação de susceptíveis é muito bai- 
xa. Alem disso, as condições climáticas do semi-árido não favorecem a sobrevivência dos parasitos no meio ambiente durante os longos períodos de seca. A resistência dos bovinos maiores de 2 anos as parasitoses gastrintestinais é uma fato comprovado no Brasil (Costa et al. 1974, Carneiro \& Freitas 1977, Charles 1992, Padilha 1996, Lima 1998, Pimentel Neto \& Fonseca 1999) e em outros países (Roberts et al. 1952, Tongson \& Balediata, 1972).

Animais jovens apresentaram maior acometimento por eimeriose (Quadro 3) o que se deve a uma ausência de imunidade desta categoria frente à infecção. Nesta idade a enfermidade é de propagação rápida e caracteriza-se por causar, principalmente diarréia. Tanto em ovinos quanto em caprinos, a eimeriose é uma doença de animais confinados ou semi-confinados e a freqüência da mesma no semi-árido deve-se ao fato de que os pequenos ruminantes, mesmo em condições de cria extensiva, permanecem à noite em capris ou ovis com alta lotação de animais de diversas idades, facilitando a transmissão.

Para a tristeza parasitária, o semi-árido apresenta áreas de estabilidade enzoótica como Campina Grande e outras de instabilidade como Boqueirão e Carirí (Madruga et al. 1993). Não há dados em relação ao sertão, mais a ocorrência de surtos de tristeza parasitária indica que esta região é também de instabilidade enzoótica. Os surtos ocorrem no final da época de chuvas nas áreas de planaltos e serras da região da Borborema, com alturas superiores a $400 \mathrm{~m}$, em áreas úmidas como a bacia do Rio do Peixe e Rio Piranhas e também em áreas irrigadas, como no município de Patos, em que há a formação de microclimas favoráveis à sobrevivência do carrapato. Ainda se observam surtos em áreas mais secas e de menor altitude (200-400m) do alto sertão, onde o carrapato não sobrevive à seca. Estes ocorrem quando animais com carrapato são introduzidos no início da chuva e o parasita consegue se multiplicar causando surtos de tristeza parasitária no final da chuva ou início da seca. Como ocorre em outras áreas de instabilidade enzoótica (Mahoney \& Ross 1972, Farias 2007) a categoria mais afetada no presente estudo foram os animais adultos (2-6 anos). Para estabelecer medidas corretas de controle de Rhipicephalus (Boophilus) microplus e tristeza parasitária, na região, são necessários trabalhos de pesquisa sobre ecologia (dinâmica de população) do parasita nas diferentes regiões do sertão, assim como da prevalência dos diferentes agentes da tristeza parasitária.

\section{REFERÊNCIAS}

ADENE, Agência de Desenvolvimento do Nordeste. Acesso em: 15 de julho de 2007. http:// www.adene.gov.br

Arosemena N.A.E., Bevilaqua C.M.L., Melo A.C.F.L. \& Girão M.D. 1999. Seasonal variations of gastrointestinal nematodes in sheep and goats from semi- arid area in Brazil. Revta Med. Vet. 150:873-876.

Athayde A.C.R., Nunes R., Araújo M.M. \& Silva W.W. 1996. Surto epizoótico de haemoncose e estrongiloidose caprina no semi-árido paraibano. Anais XV Congresso Panamericano de Ciências Veterinárias, Campo Grande, MS, p.264.

Carneiro J.R. \& Freitas M.G. 1977. Curso natural de infecções helmín- ticas gastrintestinais em bezerros nascidos durante a estação chuvosa em Goiás. Arq. Esc. Vet., Belo Horizonte, 29(1):49-62.

Charles T.P. 1992. Verminoses dos bovinos de leite, p.55-110. In: Charles T.P. \& Furlong J. (Eds), Doenças Parasitárias dos Bovinos de Leite. Embrapa-CPGL, Coronel Pacheco, MG.

Charles T.P. 1989. Seasonal prevalence of gastrointestinal nematodes of goats in Pernambuco state, Brazil. Vet. Parasitol. 30:335-343.

Costa V.M.M. 2008. Comunicação pessoal (Universidade Federal de Campina Grande, Campus de Patos, PB).

Costa E.A. \& Vieira L.S. 1984. Evolução do parasitismo por nematódeos gastrintestinais em caprinos no sertão dos Inhamus, Ceará. Pesquisa em Andamento no.9, Embrapa-CNPCO, Sobral, p.1-4.

Costa H.M.A., Guimarães M.P., Costa J.O. \& Freitas M.G. 1974. Variação estacional da intensidade de infecção por helmintos parasitos de bezerros em algumas áreas de produção leiteira em Minas Gerais, Arq. Esc. Vet. UFMG, Belo Horizonte, 26(1):143-153.

Costa Júnior G.S., Mendonça I.V., Campelo J.E.G., Cavalcante R.R., Dantas Filho L.A., Nascimento I.M.R., Almeida E.C.R. \& Chaves R.M. 2005. Efeito de vermifugação estratégica, com princípio ativo à base de ivermectina na incidência de parasitos gastrintestinais no rebanho caprino da UFPI. Ciênc. Anim. Bras. 6 (4):279-286.

CSIRO 1994. Successful worm treatment. Folder, Division of Animal Health, Communication Group, Commonwealth Scientific and Industrial Research Organization, Australia.

Farias N.A. 2007. Tristeza parasitária, p.524-532. In: Riet-Correa F., Schild A.L., Lemos R.A.A. \& Borges J.R.J. (Eds), Doenças de Ruminantes e Eqüinos. Vol.1. 3aㅡ. ed. Pallotti, Santa Maria, RS.

Lima W.S. 1998. Seasonal infection pattern of gastrointestinal nematodes of beef cattle in Minas Gerais State, Brazil. Vet. Parasitol. 74(2/4):203214,

Macêdo J.T.S.A., Riet-Correa F., Dantas A.F.M. \& Simões S.V.D. 2008. Doenças da pele em caprinos e ovinos no semi-árido. Pesq. Vet. Bras. 28(12):633-642,

Madruga C.R., Aycardi E., Kesller R.M., Schenk M.A.M., Figueiredo G.R. \& Curvo J.B.E. 1984. Níveis de anticorpos anti-Babesia bigemina e Babesia bovis em bezerros da raça Nelore, Ibagé e cruzamentos de Nelore. Pesq. Agropec. Bras. 19:1163-1168.

Mahoney D.F. \& Ross D.R. 1972. Epizootiological factors in the control of bovine babesiosis. Aust. Vet. J. 48(5):292-298.

Molento M.B. 2005. Avanços no diagnóstico e controle das helmintoses em caprinos. I Simpósio Paulista de Caprinocultura (SIMPAC). Multipress, Jaboticabal, p.101-110.

Padilha T. 1996. Estratégia para o controle da verminose gastrintestinal de bovinos de leite na região sudeste do Brasil. Anais I Simpósio de Controle de Parasitos, Campinas, p.57. (Resumo)

Pereira M.C., Labruna M.B., Szabó M.P.J. \& Klafke G.M. 2008. Rhipicephalus (Boophilus) microplus: biologia, controle e resistência. Med. Vet., São Paulo. 169p.

Pimentel Neto M. \& Fonseca A.H. 1999. Epidemiologia das helmintoses pulmonares e gastrintestinais de bovinos de leite na microrregião homogênea do Vale do Paraíba Fluminenese. Hora Vet., Porto Alegre, 19(112):41-46.

Ruas J.L. \& Berne M.E.A. 2001. Parasitoses por nematódeos gastrintestinais em bovinos e ovinos, p.19-162. In: Correa F.R., Schild A.L., Mendez M. del C. \& Lemos R.A.A. (Eds), Doenças de Ruminantes e Eqüinos. Vol.2. $2^{\text {a }}$ ed. Varela, São Paulo. 573p.

Roberts F.H.S., O'Sullivan P.J. \& Rieck R.F. 1952. The epidemiology of parasitic gastro- enteritis of cattlle. Aust. J. Agric. Res. 4(3):11871226.

Rodrigues A.B., Athayde A.C.R., Rodrigues O.G., Silva W.W. \& Faria E.B. 2007. Sensibilidade dos nematóides gastrintestinais de caprinos e ovinos a anti-helmínticos na mesorregião do sertão paraibano. Pesq. Vet. Bras. 27(4):162-166. 
Rosa J.S. 1996. Enfermidades em caprinos: diagnóstico, patogenia, terapêutica e controle. Embrapa Caprinos, Sobral, CE. 196p.

Tongson M.S. \& Balediata E. 1972. Epidemiology of bovine parasitic gastroenteritis. J. Vet. Med., Berlin, 11:63-72.

Torres-Acosta J.F.J. \& Hoste H. 2008. Alternative or improved methods to limit gastro-intestinal parasitism in grazing sheep and goats. Small Rum. Res. 77:159-173.

Vieira L.S. 2003. Alternativas de controle da verminose gastrintestinal dos pequenos ruminantes. Circular Técnica, Embrapa Caprinos, Sobral.10p.

Vieira L.S. 1999. Epidemiologia e controle da nematodeose gastrin- testinal dos caprinos. Anais Congresso Pernambucano de Medicina Veterinária. Sociedade Pernambucana de Medicina Veterinária, Recife, p.123-128.

Vieira L.S., Cavalcante A.C.R. \& Ximenes L.J.F. 1997. Epidemiologia e controle das principais parasitoses de caprinos nas regiões semiáridas do Nordeste do Brasil. Circular Técnica, Embrapa Caprinos, Sobral, CE. 49p.

Vieira L.S., Berne M.E.A. \& Costa C.A.F. 2007. Parasitoses por nematódeos gastrintestinais em caprinos, p.604-616. In: Riet-Correa F., Schild A.L., Lemos R.A.A. \& Borges J.R.J. (Eds), Doenças de Ruminantes e Eqüinos. Vol.1. $3^{a}$ ed. Pallotti, Santa Maria, RS. 